\title{
ON SPECTRA OF HAUSDORFF OPERATORS ON $l_{+}^{2}$
}

\author{
JAMES A. DEDDENS ${ }^{1}$
}

\begin{abstract}
In this note we compute the spectra of all Hausdorff operators on $l_{+}^{2}$. We also compute the norm and point spectra of the adjoint. Our technique uses standard operator theory and analytic function theory.
\end{abstract}

Several results concerning the norm and spectrum of Hausdorff matrices on $l_{+}^{p}$ have been obtained by B. E. Rhoades [6]. In this note we give an alternate approach to these results on $l_{+}^{2}$. Our technique involves standard operator theory and the use of analytic functions, and is in the spirit of the classical paper on the subject [4]. That analytic functions play a key role in the study of Hausdorff matrices has been previously noted [4]. We feel that our approach is less computational and gives a better understanding of why the spectrum is what it is.

We want to thank Professor B. E. Rhoades for several discussions regarding the contents of this note, and Professor A. Shields for pointing out the relevance of [7] to our Theorem. Related results also appear in [9].

Let $\left\{\mu_{k}\right\}_{k=0}^{\infty}$ be a sequence of complex numbers, and let $\Delta$ be the forward difference operator defined by $\Delta \mu_{k}=\mu_{k}-\mu_{k+1}, \Delta^{n} \mu_{k}=\Delta\left(\Delta^{n-1} \mu_{k}\right)$ for $k=$ $0,1,2,3, \ldots$ and $n=1,2,3, \ldots$ A Hausdorff matrix $H$ is a matrix whose entries are given by $H_{n k}=\left(\begin{array}{c}n \\ k\end{array}\right) \Delta^{n-k} \mu_{k}$ if $k \leqslant n$ and $H_{n k}=0$ if $k>n$. A Hausdorff operator is a bounded linear operator on $l_{+}^{2}$ whose matrix with respect to the usual basis is a Hausdorff matrix. Some of the well-known Hausdorff operators and their generating sequences are: Cesàro, $C_{\alpha}, \mu_{n}=$ $\Gamma(\alpha+1) \Gamma(n+1) / \Gamma(n+\alpha+1)$; Hölder, $H_{\alpha}, \mu_{n}=(n+1)^{-\alpha}$; Euler, $(E, r)$, $\mu_{n}=a^{n}$ where $r=(1-a) / a$; Gamma, $\Gamma_{a}^{\alpha}, \mu_{n}=(a /(n+a))^{\alpha}$; and Generalized Cesàro, $C_{a}^{\alpha}, \mu_{n}=\Gamma(a+\alpha) \Gamma(n+a) / \Gamma(a) \Gamma(n+a+\alpha)$. In all cases we must have $a \geqslant \frac{1}{2}$.

The key fact needed to compute the norm and spectrum of a Hausdorff operator is that every Hausdorff operator commutes with the Cesàro operator $C_{1}$ of order 1 [3], [4]. A. Shields and L. Wallen [7] have shown that the commutant of $C_{1}$ (denoted by $C_{0}$ by Shields and Wallen) is exactly the set of operators which are bounded analytic functions of the operator $C_{1}$. Thus the spectral theorem can then be used to compute the spectrum [2]. It is also known [5] that $C_{1}$ is a subnormal operator with a cyclic vector, and hence [8] each Hausdorff operator is subnormal. But subnormal operators satisfy:

Received by the editors July 6, 1977.

AMS (MOS) subject classifications (1970). Primary 40C15, 40G05, 47A15.

Key words and phrases. Hausdorff matrices, Cesàro operator, spectrum, norm.

${ }^{1}$ Research supported by NSF Grant MCS 77-01850. 
norm equals spectral radius [2]. Thus the norm of a Hausdorff operator is merely the maximum of the moduli of the elements in its spectrum. Since the adjoint of $C_{1}$ has an open disk of eigenvalues [5], the adjoint of every Hausdorff operator, being a function of $C_{1}^{*}$, also has a nonempty open set of eigenvalues (compare this with Theorem 3 in [6]). We summarize these results in the following theorem:

THEOREM. If $H$ is a Hausdorff operator on $l_{+}^{2}$ then there exists a bounded analytic function $\psi$ on $E=\{z \in \mathbf{C}:|z-1|<1\}$, with $\psi(1)=1$, such that $H=\psi\left(C_{1}\right)$. Then $\psi(E)$ is a nonempty open set, $\sigma(H)=$ closure $\psi(E)$, and the point spectrum $\sigma_{p}\left(H^{*}\right)$ contains the set $\psi(E)^{-}$where - denotes complex conjugation. Finally $\|H\|=\sup \{|\lambda|: \lambda \in \psi(E)\}$.

Thus the only difficulty in computing the norm and spectrum of a Hausdorff operator $H$ is in determining the bounded analytic function that yields $H$. Actually this turns out to be quite simple: by considering the matrices for $H$ and $\psi\left(C_{1}\right)$ one establishes that $\mu_{n}=\psi(1 /(n+1))$. This determines $\psi$ as long as $\mu_{(1 / z)-1}$ is analytic at 0 . Namely $\psi(z)=\mu_{(1 / z)-1}$. We now proceed to carry out this calculation for the above-mentioned Hausdorff operators.

CESÀro OPERATOR, $C_{\alpha}$, here $\psi_{\alpha}(z)=\Gamma(\alpha+1) \Gamma(1 / z) / \Gamma(1 / z+\alpha)$. So $\sigma\left(C_{\alpha}\right)=$ closure $\psi_{\alpha}(E)$ and, it is clear that

$$
\left\|C_{\alpha}\right\|=\sup \left\{|\lambda|: \lambda \in \psi_{\alpha}(E)\right\}=\psi_{\alpha}(2)=\Gamma(\alpha+1) \Gamma\left(\frac{1}{2}\right) / \Gamma(\alpha+1 / 2) .
$$

HölDER OPERATOR, $H_{\alpha}$, here $\psi_{\alpha}(z)=z^{\alpha}$. So

$$
\sigma\left(H_{\alpha}\right)=\left\{z^{\alpha}:|z-1| \leqslant 1\right\} \quad \text { and } \quad\left\|H_{\alpha}\right\|=\psi_{\alpha}(2)=2^{\alpha} .
$$

EULER OPERATOR, $(E, r)$, here $\psi_{r}(z)=a^{(1 / z)-1}$. So

$$
\sigma((E, r))=\left\{a^{(1 / z)-1}:|z-1| \leqslant 1\right\}=\left\{z:|z| \leqslant(1+r)^{1 / 2}\right\} .
$$

Hence $\|(E, r)\|=(1+r)^{1 / 2}$. This case was previously studied in [1].

GAMMA OPERATOR, $\Gamma_{a}^{\alpha}$, here

$$
\psi_{a}^{\alpha}(z)=[(1 / z-1+a) / a]^{-\alpha}=\left(\frac{z a}{1+(a-1) z}\right)^{\alpha}
$$

So

$$
\sigma\left(\Gamma_{a}^{\alpha}\right)=\left\{\left(\frac{z a}{1+(a-1) z}\right)^{\alpha}:|z-1| \leqslant 1\right\}
$$

and

$$
\left\|\Gamma_{a}^{\alpha}\right\|=(a /(a-1 / 2))^{\alpha} .
$$

In case $\alpha=1$, then $\psi_{a}^{1}$ is a linear fractional transformation, and hence takes circles to circles. Hence $\sigma\left(\Gamma_{a}^{1}\right)$ is a closed disk, in fact

$$
\sigma\left(\Gamma_{a}^{l}\right)=\left\{z:\left|z-\frac{a}{2 a-1}\right| \leqslant \frac{a}{2 a-1}\right\}
$$


This explains Theorem 4 in [6] and the remarks preceding it. In fact, this is about the only time when $\sigma(H)$ is a closed disk.

Generalized Cesàro operator, $C_{a}^{\alpha}$, here

$$
\psi_{a}^{\alpha}(z)=\Gamma(a+\alpha) \Gamma(1 / z-1+a) / \Gamma(a) \Gamma(1 / z-1+a+\alpha) .
$$

So again $\sigma\left(C_{a}^{\alpha}\right)=$ closure $\psi_{a}^{\alpha}(E)$ and it is easily seen that $\left\|C_{a}^{\alpha}\right\|=\psi_{a}^{\alpha}(2)=$ $\Gamma(a+\alpha) \Gamma(a-1 / 2) / \Gamma(a) \Gamma(a+\alpha+1 / 2)$.

\section{REFERENCES}

1. J. A. Deddens, Analytic Toeplitz and composition operators, Canad. J. Math. 24 (1972), 859-865.

2. P. R. Halmos, Hilbert space problem book, Van Nostrand, Princeton, N. J., 1967.

3. F. Hausdorff, Summationsmethoden und momentfolgen. I, Math Z. 9 (1921), 74-109.

4. W. A. Hurwitz and L. L. Silverman, On the consistency and equivalence of certain definitions of summability, Trans. Amer. Math. Soc. 18 (1917), 1-20.

5. T. L. Kriete and D. Trutt, The Cesaro operator in $l^{2}$ is subnormal, Amer. J. Math. 93 (1971), 215-225.

6. B. E. Rhoades, Spectra of some Hausdorff operators, Acta Sci. Math. (Szeged) 32 (1971), 91-100.

7. A. L. Shields and L. J. Wallen, The commutants of certain Hilbert space operators, Indiana Univ. Math. J. 20 (1971), 777-788.

8. T. Yoshino, Subnormal operator with a cyclic vector, Tôhoku Math. J. 21 (1969), 49-55.

9. G. Leibowitz, Discrete Hausdorff transformations, Proc. Amer. Math. Soc. 38 (1973), 541-544.

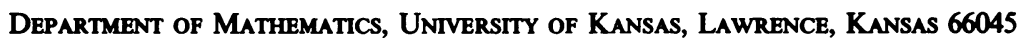

Current address: Department of Mathematics, University of Cincinnati, Cincinnati, Ohio 45215 